\title{
THE DEVELOPMENT OF CAMERA CALIBRATION METHODS AND MODELS
}

\author{
By T. A. CLARKE ${ }^{1}$ and J. G. FRYER ${ }^{2}$
}

1. Department of Electrical, Electronic, and Information Engineering, City University, London, UK. 2. Department of Civil Engineering and Surveying, The University of Newcastle, Australia.

\begin{abstract}
Correction for image distortion in cameras has been an important topic for as long as users have wanted to faithfully reproduce or use observed information. Initially the main application was mapping. While this task continues today other applications also require precise calibration of cameras such as close range 3-D measurement and many 2-D measurement tasks. In the past the cameras used were few in number and highly expensive whereas today a typical large industrial company will have many inexpensive cameras being used for highly important measurement tasks. Cameras are used more today than they ever were but the golden age of camera calibration for aerial mapping is now well in the past. This paper considers some of the key developments and attempts to put them into perspective. In particular the driving forces behind each improvement have been highlighted.
\end{abstract}

\section{INTRODUCTION}

A camera consists of a image plane and a lens which provides a transformation between object space and image space. This transformation cannot be described perfectly by a perspective transformation because of distortions which occur between points on the object and the location of the images of those points. These distortions can be modelled. However, the model may only be an approximation to the real relationship. How closely the model conforms to reality will depend on the model and how well the model's parameters can be estimated. Choosing parameters which are both necessary and sufficient has taxed those involved in the process of lens calibration for as long as lenses have been used to make precise measurements.

In recent years lens calibration has received less attention than in the period from 1950-1970. This may be attributed to the maturity of understanding of aerial lenses which in the past provided much of the stimulus for the development of models and calibration methods. Also, the widespread use of self calibration using bundle adjustment methods has meant that a high level of performance has become commonplace. It may be argued that the current static situation means that there is no further research necessary. However, the development of camera calibration methods and models has always been interspersed with periods of relative stability. The demand for high-speed continuous measurement using large sensors arrays may require new advances to be made.

This paper considers lens calibration methods for close range photogrammetric purposes with the objective of analysing whether there are lessons to be learnt from past research and development. It is hoped that the paper will provide a good starting point for those who wish to conduct research into lens calibration for close-range photogrammetry. A listing of 91 articles on aspects of camera calibration for the period 1889 until 1951 is provided by Roelofs (1951). Some of these articles are briefly referenced in the following sections, although the emphasis here is more on the development of models for lens distortion and the evolution of methods to measure and account for that distortion.

\section{STEREOSCOPIC MAPPING}

The earliest uses for photogrammetry were in mapping. The procedures used were more qualitative than quantitative, and the equipment took little account of the problems associated with geometric errors introduced to the images by the camera. Aerial photogrammetric mapping had to wait until the development of reliable aircraft in the era of World War I. Before this time terrestrial cameras 
were used to carry out some mapping operations. It is worth noting the pioneering camera calibration work of Deville in Canada (Field, 1946) who had established a laboratory in 1910 with collimators to calibrate components of his 'surveying camera', along with other equipment such as levels and transits.

Experiences during World War 1 had demonstrated the benefits of aerial surveying and, linked with developments in early stereoscopic plotting instruments, it soon became obvious that to achieve higher accuracies in stereo photogrammetric measurements that some knowledge or calibration of the lens system was necessary. The first aerial camera to be calibrated in Canada was in 1920 and the important constants determined were the principal distance and the location of the principal point. An autocollimation method was employed to discover the principal point. A geodetic theodolite was used to observe the angles through the lens to a grid plate containing finely etched crosses to compute values for the principal distance. By examining the range of principal distances computed along one or more radials along the image plane, a 'calibrated' value was selected to minimise the average distortion. For the next thirty years, the techniques employed in Canada were basically the same, with refinements occurring naturally as standards of measuring equipment improved. The techniques have been given the generic title of 'visual calibrations', as opposed to 'photographic calibrations' which were to assume preeminence from the 1950's.

In the USA, government agencies started to submit cameras to the National Bureau of Standards for calibration shortly after World War I. Visual optical benches were first used with results sufficiently precise for the requirements of aerial photogrammetry at that time (Washer, 1957b). In the late 1930's a precision lens testing camera was developed at the US Bureau of Standards and employed on camera calibration (Gardner and Case, 1937). By the late 1940's, the volume of work had grown and there was a need for greater accuracy and easier-to-operate equipment. A camera collimator based on a bank of 25 collimators arranged in the form of a cross (six collimators on each arm symmetric to a central collimator) was designed (Washer and Case, 1950).

For mapping applications the earliest solutions to the problems associated with large radial lens distortions were by direct optical correction whereby the image was re-projected through the camera and lens system which had captured it. This system was termed the Porro-Koppe Principle after the scientists who perfected it in the latter part of the 19th century. In this manner the geometric distortions in the image were cancelled. Similarly, in the Multiplex system, the original aerial photograph captured with a Metrogon lens was re-projected through a reduction lens system which corresponded with the distortion values for a nominal Metrogon lens. This provided a small $\left(50 \mathrm{~mm}^{2}\right)$ transparency which was then used in the projector system for stereo viewing and plotting (Baker, 1980). Other correction mechanisms employed included the use of a distortion-correction cam in the Kelsh plotter to vary the equivalent focal length, and the use of aspheric distortion-compensating plates in stereoplotters such as the Wild Autograph. Numerous varieties of correction curves and graphs were compiled to correct parallax values or elevations at any given point in stereomodels. It was noted that their application became "very tedious" (Bean, 1940).

The Second World War caused a dramatic increase in the use of aerial photography for reconnaissance and mapping so that by the late 1940's there was international recognition that some standardisation of techniques for camera calibration would be beneficial. Mapping for military purposes was a driving force (Corten, 1951) and partially as a result of the 1948 ISPRS Congress, a meeting between representatives of camera calibration authorities of various European countries was held in Paris in late 1950. The results of their deliberations were discussed at a meeting of the American Society of Photogrammetry in Washington in January, 1951. Topical opinions on calibration were presented by camera manufacturers, calibration authorities and academic photogrammetrists, and reviewed in a panel discussion. Representatives from North American and European countries were present. This meeting was probably a direct consequence of the post-war mapping boom and a serious attempt at understanding the methodologies for camera calibration employed in different countries.

The relative paucity of stereo or other map-plotting equipment which large organisations such as the US Coast and Geodetic Survey held in 1950 is also relevant to this discussion. Tewinkel (1951) noted that they had one Zeiss Stereoplanigraph and a total of only seven Multiplex or Kelsh plotters for 
production purposes. He further stated "...a greater bulk of photogrammetric mapping is being performed without special instruments other than a stereoscope". The majority of the work was being done by "...radial plotting and manual graphic compilation". Radial line plotting methods are insensitive to radial distortions, but require an accurate position for the principal point. There was little use of stereo-photography for accurate heighting.

The contentious issues of the day concerned the location of the principal point (or a point where distortions were symmetric), the intersection point of the fiducial marks, and the 'calibrated' focal length (see, for example, Pestrecov, 1951). By changing the value of the focal length, a radial distortion curve which essentially changed almost linearly with radial distance, distortion errors could be reduced to an amount under $+/-20 \mu \mathrm{m}$. This new value for the focal length was termed the "calibrated focal length" and was that value which best 'balanced' out the radial distortion into equal amounts of positive and negative distortion. Hence the large (by today's norms) radial distortion did not seem to represent cause for concern, given the radial nature of much of the stereoplotting. Odle (1951) noted that "...in automatic plotting instruments a standard radial distortion is assumed and corrected for", with the uncertainty in the radial distortion curve for the new Williamson and Ross aerial survey lenses not to exceed $+/-20 \mu \mathrm{m}$. This 'standard' lens had a radial distortion curve of $160 \mu \mathrm{m}$ at a radial angle of 35 degrees from the principal point. This value agrees closely with that quoted by Pestrecov (1951) from the Bausch and Lomb Optical Co. who claimed “...the astonishingly low value of $0.1 \%$..." (equivalent to $150 \mu \mathrm{m})$ for a Metrogon lens.

The reports of their meeting provide an insight to the resolving power or imaging quality of the lens/films of the day. This is a factor which should not be overlooked in this general discussion about the evolutionary processes in lens and camera calibration. Katz (in discussion in Sanders, 1951) stated that "..it was not a simple matter of stating which of two lenses were the best simply because one could resolve 1000 lines per millimetre and another only 50. ...out in service we most frequently get 15 or 12 or lower". MacDonald (1951) also mentions 12 lines per $\mathrm{mm}$ resolution, so for general aerial photogrammetric purposes there was not much pressure on finding a better theoretical, or empirical, solution to the tangential or prism effect. However, such effects were beginning to exercise the minds of specialists at the various national calibration authorities.

Several photogrammetrists had mentioned the problems of the tangential effect after World War II. There was no method to correct for these asymmetric or tangential distortions which were sometimes termed 'decentering' (misalignment of lens components relative to the optical axis) or equivalent to a 'prism' effect (the effect arising from a thin prism placed in front of a lens, for example, Odle (1951)). Pennington (1947) discussed tangential distortion and its effect on the photogrammetric extension of control. Since slotted templates and other radial line plotting techniques were being extensively used, any effect such as tangential distortion, (which caused errors in angles based on the principal point), had to be investigated. Gardner, Chief of the Optical Instruments section of the US Bureau of Standards (1949) stated "...tangential distortion in which all photogrammetrists are now so much interested ....must be due to faulty glass or to imperfect centering of the lens components". However he noted that "...further improvements (in lens designs) will only give diminishing returns because of the differential shrinkage of the film base". He thought that once values of the lens distortions of 20 to $30 \mu \mathrm{m}$ over the entire negative were achieved, glass plates would once again have to be used but this would require more accurate calibration procedures to be devised.

Against this background of concentration on accuracy in a radial direction from the centre of the image plane, it was understandable that a meeting in 1951 raised concerns about the problems which could occur with lenses which exhibited tangential distortions (for example, Pestrecov, 1951). He concluded that these should be kept below an "acceptable standard" and their determination was encouraged, although if the tangential distortion was greater than approximately $30 \mu \mathrm{m}$ then that lens should not be used for aerial map production (see also Hothmer, 1958). Washer (1957a) suggested 15 $\mu \mathrm{m}$ as the acceptable "tolerance" for tangential distortion. 
Another facet of total camera calibration which did not receive much attention during this time, but is now known to be of primary importance concerns the flatness of the imaging media. Macdonald (1951), who was an academic representative rather than member of a national calibrating authority at the 1951 meeting, mentioned the need for " ...calibration of the lens, camera and photographic material combination ..." using the technique to be "... employed in practice". He did not single out the image platen for special mention, but clearly had a bias towards field calibration procedures over laboratory ones where the lens and camera body were often calibrated in isolation of the film backing plane. The national authorities in Canada, England and the USA did not seem to share his viewpoint and concentrated their efforts on laboratory techniques. In 1955 Carmen published a paper entitled "Control and Interferometric Measurement of Plate Flatness" and brought to wider attention the importance of this aspect of total camera calibration.

One authority which favoured 'field camera calibration' techniques was the US Naval Photographic Interpretation Centre. Merritt (1951) discussed several camera calibration techniques but indicated a strong preference for either the methods using the goniometer or star exposure. $\mathrm{He}$ suggested that all other methods such as those involving collimators "....are regarded as expedients with particular application to non-metrical cameras". Clearly the star exposure method could capture any irregularities of the film flattening or reseau plate if film were used, but mostly these large cameras used glass plates which were reasonably flat. The flatness of pressure plates for film cameras did not seem so important in 1951, probably because of the previously mentioned uncertainties in film distortion and the general level of accuracy in the calibration process.

The Seventh International Congress of Photogrammetry in 1952 adopted a Resolution of Commission 1 which suggested the calibration of cameras by photographic procedure was preferable to visual methods, although the latter could still be used if they gave the same results to within the required accuracy. Visual methods using the goniometer had more frequently been applied in Europe, whereas greater use had been made of field and collimator methods in Canada and the USA (Hothmer, 1958). Carman and Brown of the National Research Council of Canada (1956) were finding consistent differences by using photographic techniques from the visual calibration data supplied by the manufacturers. Calibrated focal lengths usually exceeded the manufacturer's values by 10 or $20 \mu \mathrm{m}$ and radial distortion values were averaging $6 \mu \mathrm{m}$ higher with peaks up to $17 \mu \mathrm{m}$. They concluded that the differences could be attributed to chromatic differences of distortion as seen by two spectrally different receivers and claimed the visual test procedure used the wrong chromatic sensitivity since the minusblue filter was usually not included in the testing. Their results emphasised the necessity of calibrating the air survey cameras by a procedure which closely simulated the conditions of use.

There was a good deal of discussion in the mid-1950's about radial distortion and its presentation in a form which would be easily understood (and not misunderstood) by those involved in the mapping process. Lewis (1956) elaborated on the nature of the radial distortion curve and how its magnitude was a direct function of the value chosen for the equivalent focal length of the camera. He argued against producing multiple radial distortion curves, each related mathematically to a particular value of the focal length. He proposed a curve of distortion based on variations of focal length instead and concluded "...that the usual method of presenting distortion data carries with it the pitfall of misinterpretation into which the ordinary user is very likely to fall". It does not seem that his recommendations were followed.

Considerable variations in calibration results for the same type of camera were noted by Hothmer (1958). He believed they were due to manufacturing limitations, especially with regard to slightly differing refractive indices in the batches of glass lenses from one production series to the next. A test of 276 Metrogon lenses showed radial distortion differences up to $50 \mu \mathrm{m}$ although $50 \%$ of all radial distortion curves fell within a $+/-10 \mu \mathrm{m}$ envelope. These tests demonstrated the size of errors which could reasonably be expected to occur from standard correction plates fitted to stereoplotting instruments. 
Hothmer (1958) also discussed the effects of variations in temperature from the calibration laboratory to those which might be expected in flight. A difference of $50{ }^{\circ} \mathrm{C}$ was likely $\left(20{ }^{\circ} \mathrm{C}\right.$ to $\left.-30{ }^{\circ} \mathrm{C}\right)$ and reported that few tests had thus far been conducted on this effect. A Topar and a Pleogon lenses had been placed "...in a big refrigerator" at $-20{ }^{\circ} \mathrm{C}$ for three hours and when tested the resulting distortion curves varied "...in the order of $+/-5 \mu \mathrm{m}$ ". Hothmer's thorough paper also investigated the flatness of suction plates and the flatness of the glass plates for photography made by Gevaert. He concluded that radial distortions from $5 \mu \mathrm{m}$ to $20 \mu \mathrm{m}$ could also be produced by these two error sources respectively and further noted that if film were to replace the glass plates, then irregular shrinkage could introduce errors of the same magnitude. In an attempt to address several of the deficiencies of laboratory tests, he reported on a practical calibration test made with photography over Swedish lakes in the Spring. They were just melting and some definition was visible on the otherwise flat surface. Stereoscopic pairs were examined and from deviations in the derived elevations of the surface, radial distortion curves were produced. Hothmer discussed this practical method of calibration and noted that although it possessed many advantages, three major disadvantages were its dependence on the accuracy of the stereoplotting instrument, the effects of film instability, and platen unflatness.

Thompson (1957) discussed the geometrical theory of the camera. His interest was in instrument design resulting in the Thompson Watts and CP1 plotters. As a result he was concerned with the camera model and defects in the construction of cameras, he states ".....any treatment ....that cannot take the constructional defects in its stride, in particular basing the theory on the model of perspective projection with axial symmetry as an essential concomitant, is not likely to prove an advantage or be free from ambiguities.". He went on to describe a method which, for the equipment intended, allowed constructional defects and errors of observation in calibration to be included.

Hallert (1963) discussed the method of least squares applied to multi-collimator camera calibration. He achieved a combined resection and camera calibration and repeated his tests with both film and glass plates. Apart from providing some statistical significance to the radial distortion curves and other parameters of camera calibration which he computed, Hallert noted a significant difference in the radial distortion curves found from film and glass plates. "This indicates that there are additional sources of this regular error in the film negatives, probably caused by lacking flatness in the supporting back of the magazine".

In the 1950's, those involved with the manufacture and calibration of aerial cameras were obviously concerned with the investigation and discussion of the characteristics of lens distortions. Other scientists / photogrammetrists were also working on this problem, but their reports were of a more confidential nature and did not reach the public domain until Brown published a series of significant papers in the 1960's. These referred to previously confidential US Air Force Missile Test Base experiments of the mid to late 1950's.

\section{Note on multi-collimator calibration}

Camera calibration using an array of collimators which are arranged in known locations is a well developed method for calibration of aerial cameras at infinity focus. The basic scheme is illustrated in Fig 1 where each collimator produces an image at infinity of an illuminated cross-hair on the image plane. 


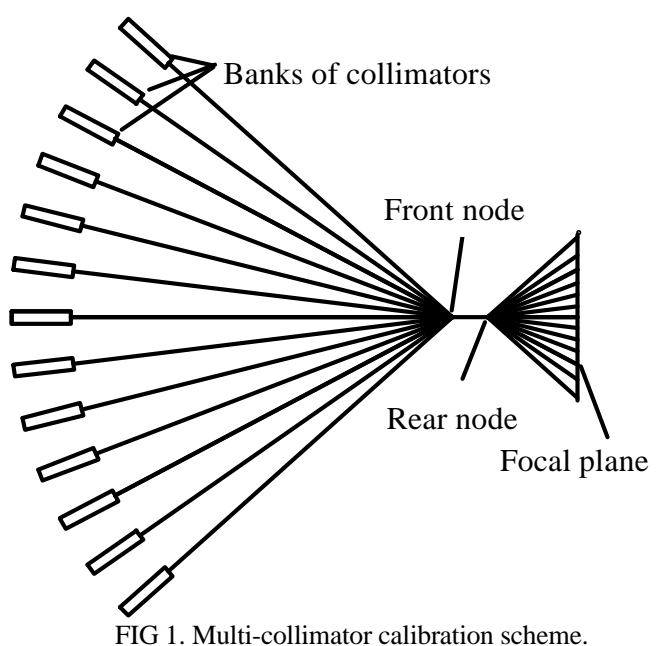

In Canada, the Canadian National Research Centre initially used a visual method from 1931 but introduced a photographic technique in 1955 as significant differences between visual and photographic methods were found. A second generation photographic method was developed by 1969 which employed collimators to produce 43 targets at infinity with an angular spacing between collimators of 90/32 degrees (Carman \& Brown, 1978; Carman, 1969). Off-axis parabolic mirrors were used to eliminate chromatic aberration. The direction defined by each collimator (aperture $63 \mathrm{~mm}$ diameter) could be considered independent of the section of the lens (defined by the camera aperture) through which the rays passed. These angles were known to within 0.5 seconds of arc in radial and tangential directions. The photographic plates were measured to a routine accuracy of $1 \mu \mathrm{m}$ at any field position. The routine calibration accuracy for $99 \%$ of measurement amounted to $\pm 3 \mu \mathrm{m}$. The US Geological survey calibrated lenses from 1953 using a multi-collimator (Karren, 1968). Their system comprised 53 collimators which were mounted in a cross formation. The camera was set up in the following way: the front node of the lens is made to coincide with the point of intersection of the 53 collimators and the focal plane was set perpendicular to the central collimator. Finally the camera was adjusted by tipping such that the plane parallel plate was perpendicular to the axis of the autocollimating telescope (Tayman, 1974).

\section{Note on the stellar calibration method}

The angular position of stars is known to a high degree of accuracy and repeatability. Shmid (1974) described the calibration of the Orbigon lens. The standard error in position of the stars was less than 0.4 seconds. Over 2420 star images were visible on each plate. A disadvantage of the method was the requirement to identify each star and apply corrections for atmospheric refraction and diurnal aberration. However, the large number of observations meant that a least squares estimation process was possible. Terms for calibrated focal length, principal point (indicated) and principal point of symmetry, radial and tangential distortion, and orientation of tangential distortion were used. The mean standard error of an observation of unit weight was about $2.7 \mu \mathrm{m}$.

\section{Note on the field calibration method}

Field calibration makes use of terrestrial features which have been surveyed to relatively high degree of accuracy to calibrate camera lenses. The advantages of the method are: in the accuracy of these points, which have typically been surveyed previously; the fact that the camera can be used in conditions similar to which it will operate; and calibration can take place at a similar time to use. A disadvantage can be the presence (for single camera calibration) or lack (for multi-camera calibration) of 3-D detail. Merrit (1948) describes a rigorous method for "determining the principal distance and the photograph co-ordinates of the plate perpendicular to the field". Other variants of this method have used a tall tower and concentric grids on the ground, and lakes which were considered acceptably flat but still had enough detail for stereo photography (Hothmer, 1958). 


\section{THE INTRODUCTION OF AN IMPROVED LENS MODEL AND SELF CALIBRATION}

In 1965 Brown presented the results of some of his earlier experiments at the Annual Convention of the American Society of Photogrammetry (later published as 'Decentering Distortion of Lenses', 1966). In this paper, Brown reviewed the scientific work of the previous 40 years which had largely equated tangential distortion to the effect obtained when placing a thin prism in front of the lens. He showed how the distortion was entirely attributable to decentering and that a rigorous analytical ray tracing paper by Conrady in 1919 could give an alternative model formulation. He stated that due to advances in analytical photogrammetric triangulation it was essential to have a lens model accurate to the limit of his comparator measurements (approximately $1 \mu \mathrm{m}$ ). Brown was almost scathing in the manner in which he stressed that “...the thin prism model be abandoned entirely... and, Conrady's model ... provides the more suitable model for decentering distortion, the validity of which in no way depends on artificial compensating motions of the plate and camera."

Brown had been involved with the photography of the trajectory of rockets against a background of stars and had used the precisely known locations of some 200 stars to calibrate a suite of ballistic cameras. As early as 1956 he had published technical reports on the simultaneous determination of lens parameters and camera orientation and had developed the bundle adjustment as a means of simultaneously solving for target co-ordinates, camera locations and lens parameters. He went on to state that since decentering and radial distortion could now be effectively modelled, there was now no impediment to using "....any well-regarded commercial lens of suitable focal length, aperture and angular field ... for it is image quality throughout the format that now becomes the overriding factor in the ultimate determination of metric potential". This statement can now be regarded as heralding a new era for non-metric photogrammetry with focussable lenses which could provide results comparable to metric cameras by using Brown's mathematical models for lens distortions.

Brown criticised the calibration techniques of the US Coast and Geodetic Survey. For example, he stated "...the ultimate effects of residual decentering distortion are accentuated by the particular reduction employed by the USCGS", and went on to explain how the calibration methods could have been improved. He further stressed that to gain the full promise of analytical photogrammetric triangulation procedures accuracies of calibration four to five times greater than those considered adequate in conventional mapping were needed. These statements must have been quite provocative at the time and indeed it was several years before his ideas gained full acceptance in the world of cartographers, mapping agencies and government authorities, many of whom would not have had the necessary awareness of computers.

\section{LEAST SQUARES APPLIED TO COLLIMATOR CALIBRATION TECHNIQUES}

In 1966, Hallert presented the results of his investigations into lens and camera calibrations in which he used the method of least squares to include redundant observations (reprinted as Hallert, 1968). He had made his observations with the latest Wild Stereocomparator StK 824 equipment at the US Geological Survey and obtained a precision as low as $0.6 \mu \mathrm{m}$. After the least squares solution, his root-mean-square (rms.) of residuals was approximately $2.5 \mu \mathrm{m}$. Similar results were obtained from least squares analyses when the new multi-collimator belonging to Wild-Heerbrugg was used to complement a series of tests of an aerial camera lens made with goniometers and collimators belonging to different European authorities. He compared the results for the radial distortion curves from the laboratory calibrations with those made from a test field in Sweden where both acetate and polyester films were used. The respective rms. values were $7.5 \mu \mathrm{m}$ and $4.5 \mu \mathrm{m}$, indicating the improvements in film technology which were occurring. Significant differences in the radial distortion curves (approximately $30 \mu \mathrm{m}$ ) were present near the edge of the format. Hallert noted these, but did not discuss them further than to suggest there may not have been enough vertical control points located near the edge of the photographs. 
At the same time that Hallert (an academic) was demonstrating the benefits of an analytical approach with redundant measurements, the Ordnance Survey in England had experimented with the use of precise theodolites for laboratory calibrations to determine radial distortion (Sly, 1968). They concluded that the use of a goniometer was a more suitable technique than those involving precise theodolites. Their requirements were for about 20 camera calibrations per year and based their decision on the fact that using a goniometer it took two persons only three hours to complete the task. This was less than half the time taken by the competing methods. Furthermore the Ordnance Survey decided to only measure two diagonals rather than all four as "... there was no significant difference in accuracy". They produced distortion corrections every $20 \mathrm{~mm}$ of radial distance using a computational technique based on graphical interpolation but noted that "....graphical methods may not be justified when electronic computing facilities are available". Tangential distortion was assumed to be negligible. In retrospect, this does not appear very enlightened considering Brown (1966) had already berated the USCGS methods and Hallert had shown several advantages from the use of redundant data. The dismissal of tangential information from the calibration process ignored the understanding of that topic which had been widely reported by other calibrating authorities since Pennington some 20 years earlier in 1947. In 1974 Hakkarainen described asymmetric radial distortion differences of up to $15 \mu \mathrm{m}$ in three Wild and Zeiss cameras which were calibrated along all four semi-diagonals by a goniometer "... with an approximate margin of $1 \mu \mathrm{m}$ " (Hakkarainen, 1974).

\section{Notes on goniometer calibration equipment and methods}

Lenses were generally calibrated at infinity focus using a collimator rotated about the front node of the lens. The principle of autocollimation was used for location of the principal point. Hallert (1960) described the goniometer principle. A precision grid is used with lines in a $10 \mathrm{~mm}$ spaced regular array. The grid is illuminated and its etched pattern projected through the lens. The illumination is normally monochromatic. A telescope, focussed to infinity, is directed towards the camera lens. The grid is projected on the collimating mark of the telescope and can be adjusted into coincidence there. By pivoting the telescope according to Fig 2 the angles can be measured. By recording the angles to selected intersection points and knowing the grid spacing, it is possible to estimate all of the camera interior orientation parameters.

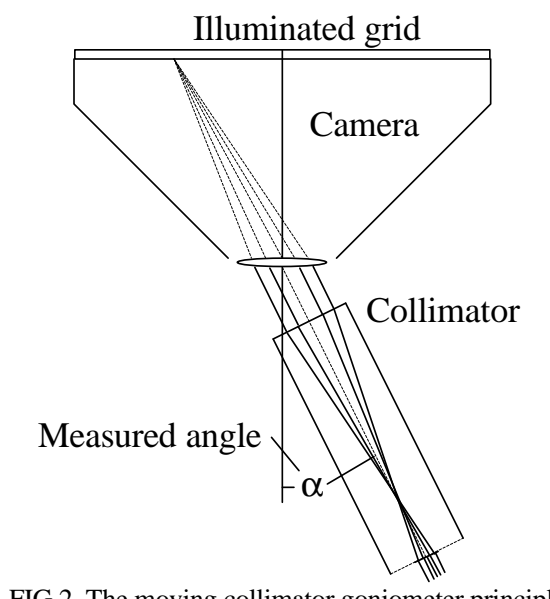

FIG 2. The moving collimator goniometer principle.

Many goniometers required the lens to be mounted with the principal axis horizontal and rotation of the camera to provide the desired two axes of rotation. An alternative goniometer configuration was similar to a theodolite in that a vertical and horizontal axis were used to measure angles about the point where two mutually perpendicular axes cross (Fig 3). 


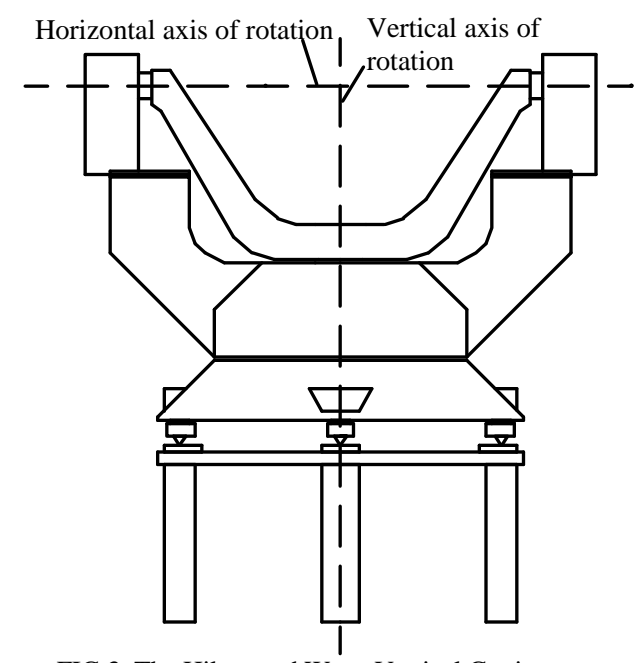

FIG 3. The Hilger and Watts Vertical Goniometer

In this system a series of mirrors was used to allow the user to stand beside the instrument and set the collimator in line with a given cross on the grid mounted in the focal plane of the lens. The angle was then read using another eyepiece.

\section{PLUMB-LINE CALIBRATION}

In 1971, Brown produced another significant paper. On this occasion he detailed the technique he had developed in which the test field consisted of a series of plumb lines. In a perspective projection, the image of a straight line will be a straight line if no lens distortions are present. Deviations in the image from straightness can be directly related to the presence of radial and decentering distortion and Brown described a mathematical model to determine the parameters of lens distortion based on the images of straight lines. The use of the word 'plumb' is interesting because although Brown initially used fine white thread stretched by plumb bobs which were stabilised by immersion in containers of oil, no use is made in his formulation of the verticality (and implied parallelism) of the string lines. Later users of this technique have photographed straight lines which have varied from the microscopic to a 10 $\mathrm{km}$ stretch of level, straight railway line in Australia for the calibration in-situ of an aerial camera (Fryer and Goodin, 1989). In his 1971 paper, Brown also revealed formulae which could accurately model radial distortion at a range of different focal length settings. If the radial distortion parameters at two separated distances (focal settings) are known, the values for all settings between them may be accurately found. Also he detailed formulae for variations of radial distortion within the photographic field of view. This is only of significance for close range camera applications. If a camera is focussed for a particular finite distance, targets at different distances will display slightly different quantities of radial distortion. In 1986 some slight modifications to similar formulae for the case of decentering distortion were published (Fryer and Brown, 1986).

The advantages of the plumbline technique are worthy of mention. The formula is reasonably simple to program on a computer and it is a practical method which does not need elaborate laboratory or field equipment. A solution is provided to the parameters of radial and decentering distortion. It is easy to collect a large number of data points and thereby obtain a reliable solution for the parameters. As an example, if the calibration of the lens of a camera is required at infinity focus, many buildings exist in most modern cities with large vertical panes of glass which can be imaged to provide a set of vertical lines. By rolling the camera through $90^{\circ}$, another image of "horizontal" lines can be taken of the same object. Estimation of the location of the imaged lines can be performed at whatever interval along the line that is desired, but approximately 30 to 50 points are regarded as sufficient. Six to ten horizontal and vertical lines are usually digitised in this manner although considerable success has been reported with as few as two lines in each orientation of the camera, provided those lines are near to the edge of the format area where the radial and decentering distortion is at its greatest (Fryer et al, 1994). The use of the plumbline technique has been reported with electro-optic (digital) cameras and automated 
measurement systems since 1986 (Fryer and Brown, 1986). It is particularly suited to such devices as line-following algorithms can obtain the required co-ordinate information without manual intervention. One disadvantage of the plumbline technique is that the offsets of the principal point from the centre of the fiducial axes system cannot easily be determined (the absence of this information can lead to significant errors in the estimation of the decentering distortion coefficients (Clarke et al, 1997)). The one exception is in the unusual case of a 'fish-eye' lens where the distortions are very large. For a determination of the offsets of the principal point, which have been shown by various researchers to be highly correlated with the parameters of decentering distortion (for example, Fryer and Fraser, 1986), a laboratory technique or the use of the self-calibrating bundle adjustment is required.

\section{ADDITIONAL PARAMETERS}

The development and use of the bundle adjustment by Brown since 1965, mostly in conjunction with terrestrial photographs (see Ziemann and El-Hakim (1982) for a brief historical development), meant that it was theoretically and practically possible to determine the parameters of lens calibration simultaneously with the determination of the three-dimensional co-ordinates of targets. This technique became known as self-calibration and is an especially strong method as all image observations, from several camera viewpoints, contribute to the determination of the unknown lens parameters. Papers dealing with self-calibration of aerial photographs began to appear in 1972 (notably, Bauer and Muller and Kenefic, Gyer and Harp). The ISPRS Congress of 1976 established a Working Group to study this technique and by 1980, Kilpela showed how the Group members had developed very many different sets of self-calibrating parameters (also known as 'additional parameters'). Many of the additional parameters appeared to have no foundations based on observable physical phenomena, but rather had been increasingly added to the mathematical model because they continued to reduce the size of the errors on the photographic plates. Brown (1972) had recognised the high correlation which existed between certain parameters and the locations and orientations of the cameras. Fraser (1982) demonstrated that the use of too many additional parameters could actually weaken the solution for the co-ordinates of target points. The over-use of additional parameters was termed 'overparameterisation'.

\section{ON-THE-JOB CALIBRATION}

The development of the bundle adjustment allowed new techniques for camera calibration to be devised. One of these was termed 'on-the-job' calibration. This is a bundle adjustment with additional parameters to describe the parameters of lens distortion, focal length, offsets of the principal point and perhaps other unknowns such as platen flatness and film shrinkage. Control points are placed in the immediate vicinity or surrounding area of the object to be imaged for the adjustment. It is the most common form of close-range camera calibration method presently being used, and is also applied by some authorities for aerial calibration using test-fields.

The term "on-the-job calibration" is sometimes confused with "self-calibration", where there is, in fact, no need for control points at all. Brown (1989) discussed the criteria which need to be met for a successful self-calibration: (1) A single camera must be used to take at least three images of the object; (2) both the interior geometry of the camera and the point to be measured on the object must remain stable during the measurement process; (3) the photogrammetric network must be strong and exercise a high degree of convergence; (4) at least one image must have a roll angle that is significantly different from the others; and (5) a relatively large number of well distributed points should be used. Given these requirements Brown comments "a satisfactory calibration of the camera can be accomplished as an integral part of the triangulation without the need for control of any kind". A difficulty with the aerial application of the self-calibrating bundle adjustment is obtaining images which have a sufficient diversity of camera angles.

\section{INTERNATIONAL STANDARDS}

By 1980 the International Organisation for Standardisation (for example see Ziemann, 1986), had consolidated its Technical Committees in the areas of photography, optics, and optical instruments to cater for the calibration requirements of lens distortions in photographic cameras. The role of a 
scientific society such as ISPRS had been clarified as one of making recommendations for procedures leading to the calibration of photogrammetric cameras and related optical tests, but not defining a standard. In the case of ISPRS, compromises in the standards for camera calibration had been on-going for over two decades. Even so, by 1986, there still existed differences in the type and extent of parameters which calibration authorities in different countries expected to be determined. In continental European countries only the parameters of interior orientation were commonly determined, whereas in North America some measure of image quality and the flatness of the focal plane was expected on a calibration certificate.

Ziemann (1986) noted a new and important distinction which had been attached to the meaning of the words 'camera calibration' as a result of non-photogrammetrists from the fields of robot or machine-vision starting to apply photogrammetric principles to new close-range tasks using cameras with CCD sensors, often the terminology "camera calibration" would only refer to the process of estimating the exterior parameters of a camera.

\section{ALGORITHMS FOR CLOSE RANGE CAMERAS}

Notwithstanding any changes of terminology, the formulae proposed by Brown back in the 1960 's appears to have remain virtually unchallenged for the past 30 years. Brown's formulations for radial and decentering distortion and for additional parameters in the bundle adjustment are described in terms of polynomials. Ziemann (1986) notes that "polynomials ... are undesirable from a mathematical point of view because of the high correlation between the different terms. It is therefore necessary to agree not only on the formulations for the lens distortion components but also on the procedure used to solve for these parameters". Ziemann proposed an algorithm for the step-by-step calibration of cameras so that the results from various calibrating authorities could be more easily compared, but it is doubted if his efforts were heeded. He suggested the order of calibration should be: the determination of rotationally-symmetric distortion; the calculation of an equivalent focal length; the determination of the decentering distortion with respect to the principal point of autocollimation; the determination of a point of best symmetry; the determination of the actual rotationally-symmetric lens distortion; and, simultaneous verification of both lens distortion components. More recently Shortis (1995) has suggested the use of the plumbline technique to determine estimates for the parameters of radial and decentering distortion, and then the use of a multi-station convergent bundle adjustment to determine the focal length and offsets of the principal point while holding the previously determined parameters of lens distortion 'fixed'. This procedure should be iterated with a re-calculation of the plumbline once the offsets of the principal point are known. This iterative, and relatively time consuming, procedure can overcome difficulties of correlation amongst parameters which might occur if the geometric strength of a self-calibration photogrammetric network is weak. If the network is strong, the need for procedures such as those described Shortis may not be necessary as a satisfactory solution to the camera and lens parameters can be obtained from the bundle adjustment.

\section{DISCUSSION AND CONCLUSIONS}

The evolution of techniques for determining lens distortions has been presented. The introduction of new models for lens distortion or methods to eliminate or negate distortion has been a gradual process as the need for increased accuracy arose. The mathematical models which describe lens distortion are essentially polynomial expressions. Care has to be exercised to ensure the correlations which are known to exist between the parameters do not lead to false answers for terms relating to, for example, decentering distortion and the offsets of the principal point. The original compensatory techniques applied to stereoplotters were analogue or mechanical in nature. Latter methods were analytical and relied on the power of computers to calculate corrections in terms of $\mu \mathrm{m}$ increments to linear encoders or stepper motors.

One interesting feature in the development of models for camera distortion has been the adoption by the modern photogrammetric community of formulae developed in the analogue and analytical periods of photogrammetry. Modern CCD or video-based cameras are physically very different 
from their analogue counterparts. An aerial lens will occupy a space of hundreds of cubic centimetres, and a lens for a digital camera may only be a few millimetres in extent, the same camera and lens distortion formulae have been used. Consider some of the important differences between film-based aerial cameras and TV-like digital cameras: the image format sizes vary from $230 \mathrm{~mm}$ by $230 \mathrm{~mm}$ to typically $6 \mathrm{~mm}$ by $4.5 \mathrm{~mm}$; the focal lengths range from $88 \mathrm{~mm}$ to $150 \mathrm{~mm}$ compared with $4.8 \mathrm{~mm}$ to 50 $\mathrm{mm}$; the fields of view from greater than $90^{\circ}$ to under $25^{\circ}$; the cost and weight of the cameras differs by a factor of approximately 1,000; the aerial camera is a purpose-built, precisely assembled piece of equipment, yet the CCD-camera is a mass production item built to satisfy a non-photogrammetric market; and, there is a fixed focus on the aerial camera, yet the video camera often permits zoom or automatic focussing.

The digital era has seen the regular use of some of the additional parameters proposed by Brown in the 1970's which don't relate specifically to lens distortions. Terms to describe the relative sizes of pixels are important, as are those which would indicate if the sensor array was tilted relative to a true image plane (trapezoidal rather than rectangular). Sensor array unflatness is becoming of concern (Fraser et al, 1995) as researchers strive to obtain ever smaller residuals after their self-calibrating bundle adjustments. A twentieth to a thirtieth of a pixel is now commonly reported as the rms. residual after adjustment, and as little as a $1 / 70$ to $1 / 100$ of a pixel has been referenced (Beyer, 1996). To achieve even lower rms. values, the past experiences detailed in this paper appear to indicate that: the camera distortion model will be further refined; target location accuracy will be improved; least squares estimation processess will be made more efficient; image processing power will increase and become more sophisticated; and camera calibration methods will be further adapted for industrial measurement tasks. For instance, over the past few years pixel sizes have become smaller while the sensors have increased in size and radiometric fidelity has improved, these trends are predicted to continue (Seitz, et al, 1995).

What does the future hold for digital photogrammetric systems in close-range environments? A current objective for real-time 3-D measurement is the determination of 3-D co-ordinates of 100-1000 points in at least $1 / 25$ of a second. Such a requirement means that each aspect of the measuring system is re-evaluated for effects such as: processing bottle-necks, ill-conditioning of equations; overparameterisation; inadequate modelling; and lack of robustness. This is of great importance if photogrammetric measuring systems are going to be used by non-experts in industrial environments and represents the challenge for photogrammetrists as the 21st century approaches. Finally this quote from the manual of photogrammetry (Slama, 1980) is worthy of repetition, "Just how much more closely the numbers can approach the "true values" depends on our knowledge of the truth. One approaches truth asymptotically, sometimes at the cost of great effort; nevertheless, it necessary to examine the path toward this ultimate goal and select reasonable limits of achievement."

\section{ACKNOWLEDGEMENTS}

The authors wish to acknowledge the contribution of Mr. K.B. Atkinson and Mr. D.A. Tait for the provision of some of the reference publications.

\section{REFERENCES}

Baker, J.G., 1980. Elements of photogrammetric optics. Manual of photogrammetry, Fourth edition, Pub. America Society of Photogrammetry. 1056 pages :103-185.

Bean, R.K., 1940. Source and Correction of Errors affecting Multiplex mapping. Photogrammetric Engineering, 6(2): 63-84.

Beyer, H.A. Uffenkamp, V. and van der Vlugt, G., 1996. Quality control in industry with digital photogrammetry. Optical 3-D Measurement Techniques III. Ed. Kahmen, H \& Grün, A. Published by Wichmann Verlag, Karlsruhe. pp. 29-38.

Brown, D.C., 1966. Decentering distortion of lenses. Photogrammetric Engineering, 32(3): 444-462. 
Brown, D.C., 1972. Calibration of close-range cameras. International Archives of Photogrammetry and Remote Sensing, 19(5) unbound paper: 26 pages, ISP Congress, Ottawa

Brown, D., 1989. A strategy for multi-camera on-the-job self-calibration. Institut Fur Photogrammetrrie Stuttgart, Festschrift, Friedrich Ackermann, zum 60. Geburtstag. 13 pages.

Burner, A.W., 1995. Zoom lens calibration for wind tunnel measurements. SPIE Vol. 2598: 19-33.

Carman, P.D. and H. Brown, 1956. Differences between visual and photographic calibration of air survey cameras. Photogrammetric Engineering. 22(4): 623.

Carman, P.D., 1969. Camera calibration laboratory at N.R.C. Photogrammetric Engineering, 35(4): 372-376.

Carman, P.D. and Brown, H., 1978. The NRC camera calibrator. Photogrammetria, 34(4): 147-165..

Clarke, T.A. Fryer, J.G. and Wang, X., 1997. The principal point for CCD cameras. Submitted to the Photogrammetric Record.

Corten, F.L., 1951. European point of view on Standardising the methods of testing photogrammetric cameras, Photogrammetric Engineering, 27(3): 401-405.

Field, R.H., 1946. The calibration of air cameras in Canada. Photogrammetric Engineering, 12(2): 142146.

Fraser, C.S., 1982. On the use of non-metric cameras in analytical non-metric photogrammetry. International Archives of Photogrammetry and Remote Sensing, 24(5): 156-166.

Fraser, C.S. Shortis, M.R. and Ganci, G., 1995. Multi-sensor system self-calibration. SPIE Vol. 2598: 218.

Fryer, J.G and Fraser, C.S., 1986. On the calibration of underwater cameras. Photogrammetric Record. 12(67): 73-85.

Fryer, J.G. and Brown, D.C., 1986. Lens distortion in close range photogrammetry. Photogrammetric Engineering and Remote Sensing, 52(2):51-58.

Fryer, J.G. and Goodin, D.J., 1989. In-flight aerial camera calibration from photography of linear features. Photogrammetric Engineering and Remote Sensing, 55(12): 1751-1754.

Fryer, J.G. Clarke, T.A. \& Chen, J., 1994. Lens distortion for simple 'C' mount lenses", International Archives of Photogrammetry and Remote Sensing, 30(5): 97-101.

Gardener, I.C. and Case 1937. Precision camera for testing lenses, Journal of Research. National Bureau of Standards, RP 984.

Gardener, I.C., 1949. New developments in photogrammetric lenses. Photogrammetric Engineering, 15(1): $38-50$

Hakkarainen, J., 1974. Determination of radial and tangential distortion of aerial cameras with a horizontal goniometer. Photogrammetric Record, 8(44): 180-187.

Hallert, B., 1960. Photogrammetry, basic principles and general survey. McGraw-Hill Book company, USA. 340 pages. 
Hallert, B., 1963. The method of least squares applied to multicollimator camera calibration. Photogrammetric Engineering, 29(5): 836-840.

Hallert, B., 1968. Notes on calibration of cameras and photographs in photogrammetry. Photogrametria, (23): 163-178.

Hothmer, J., 1958. Possibilities and limitations for elimination of distortion in aerial photographs. Photogrammetric Record. 2(12): 426-445.

Hothmer, J., 1959. Possibilities and limitations for elimination of distortion in aerial photographs (continued). Photogrammetric Record, 3(13): 60-78

Karren, R.J., 1968. Camera calibration by the multicollimator method. Photogrammetric Engineering, 34(7): 706-719.

Kenefick, J.F., Gyer, M.S. and Harp, B.F.,1972. Analytical self-calibration. Photogrammetric Engineering, 38(11):1117-1126.

Lewis, J.G., 1956. A new look at lens distortion. Photogrammetric Engineering, 22(4): 66-673.

Macdonand, D.E., 1951. Calibration of survey cameras and lens testing. Photogrammetric Engineering, 17(3): 383-389.

Merrit, E.L., 1948. Field camera calibration. Photogrammetric Engineering, 14(2): 303-309.

Merrit, E.L., 1951. Methods of field camera calibration. Photogrammetric Engineering, 17(4): 611-535.

Odle, J.E., 1951. English viewpoint: lens testing and camera calibration. Photogrammetric Engineering, 17(3): 406-412.

Pennington, J.T., 1947. Tangential Distortion and its Effects on Photogrammetric Extension of Control. Photogrammetric Engineering, 13(3): 374-385.

Pestrecov, K., 1951. Calibration of lenses and cameras. Photogrammetric Engineering, 17(3): 398-400.

Roelofs, R., 1951. Distortion, principal point, point of symmetry and calibrated principal point. Photogrammetria, 7(2): 49-66.

Sanders, R.G., 1951. A camera manufacturer's comment on camera calibration. Photogrammetric Engineering, 17(3): 415-419.

Seitz, P. Vietze, O. and Sprig, T., 1995. From pixels to answers - recent developments and trends in electronic imaging. Proc. ISPRS, 30(5W1): pp. 2-12.

Schmid, H.H., 1974. Stellar calibration of the orbigon lens. Photogrammetric Engineering, 40(1): 101111.

Shortis, M.R. Snow, W.L. Goad, W.K., 1995. Comparative geometric tests of industrial and scientific CCD cameras using plumb line and test range calibrations. International Archives of Photogrammetry and Remote Sensing, 30(5W1): 53-59.

Slama, C.C. (Editor), 1980. Manual of photogrammetry. 4th Edition. American Society of Photogrammetry, Falls Church, Virginia. 1056 pages.

Sly, W.E., 1968. The calibration of Aerial survey cameras. Photogrammetric Record, 6(31): 59-74. 
Tayman, W.P., 1974. Calibration of lenses and cameras at the USGS. Photogrammetric Engineering, 40(11): 1331-1334.

Tewinkel, G.C., 1951. Stereoscopic plotting instruments. Photogrammetric Engineering, 17(4): 635638.

Thompson, E.H., 1957. The geometrical theory of the camera and its application to photogrammetry. Photogrammetric Record, 2(10): 241-263.

Washer, F.E. \& Case, 1950. Calibration of precision airplane mapping cameras. Photogrammetric Engineering, 16(4): 502-524.

Washer, F.E., 1957a. Prism effect, camera tipping, and tangential distortion. Photogrammetric Engineering, 23(3): 721-732.

Washer, F.E., 1957b. Calibration of airplane cameras. Photogrammetric Engineering, 23(5): 890-891.

Ziemann, H. \& El Hakim, S.F., 1982. On the definition of lens distortion reference data with odd power polynomials. The International Archives of Photogrammetry, 24(1): 123-130.

Ziemann, H., 1986. Thoughts on a standard algorithm for camera calibration. Progress in Imaging Sensors, Proc. ISPRS Symposium, Stuttgart, : 41-48. 


\section{PAPER REFERENCE}

Clarke, T.A. \& Fryer, J.F. 1998. The development of camera calibration methods and models, Photogrammetric Record, 16(91): pp 51-66. 